\title{
The Computation of the Fundamental Unit of Totally Complex Quartic Orders
}

\author{
By Johannes Buchmann*
}

Dedicated to Professor Daniel Shanks on the occasion of his 70 th birthday

\begin{abstract}
We describe an efficient algorithm for the computation of the regulator and a fundamental unit of an arbitrary totally complex quartic order. We analyze its complexity and we present tables with computational results for the orders $\mathbf{Z}[\sqrt[4]{-d}], 1 \leqslant d \leqslant 500$.
\end{abstract}

1. Introduction. The computation of fundamental units in orders of algebraic number fields is one of the main problems in computational algebraic number theory.

The simplest fields for this problem are those with only one fundamental unit. There are three types of such fields; the real quadratic, the complex cubic, and the totally complex quartic fields.

It is well known that the fundamental unit of a real quadratic field can be computed by means of the ordinary continued fraction algorithm, cf. [2, II, Section 7]. There are interesting refinements of this algorithm due to Shanks [16] and Lenstra [13].

The fundamental unit of complex cubic fields can be computed using Voronoi's generalized continued fraction algorithm, cf. [5], [18]. This algorithm was discussed and improved in several interesting papers of Williams et al., cf. [20].

For totally complex quartic fields there are only a few results. If the field contains a real quadratic subfield, the computation of a fundamental unit can be reduced to the computation of the fundamental unit of the subfield, cf. [8].

For complex quartic fields containing an imaginary quadratic subfield, Scharlau proved that a fundamental unit is a minimal solution of a certain relative Pell equation, cf. [15], but he did not give a method for solving it.

For complex quartic fields containing quadratic subfields of class number one, there are results due to Amara [1] and Lakein [9], [10].

More generally, the author proved that the generalized Voronoi algorithm (GVA), developed in [3], yields a fundamental unit for any order of a totally complex quartic field. The algorithm of Jeans [7] seems to have some similarities with this method.

Received June 13, 1985; revised June 11, 1986.

1980 Mathematics Subject Classification. Primary 12A30, 12C20.

*Supported by a Feodor Lynen research fellowship of the Alexander von Humboldt-Foundation.

Permanent address: Mathematisches Institut, Universität Düsseldorf, 4000 Düsseldorf, West Germany. 
In this paper we describe how to apply the GVA practically. We analyze its complexity and prove that it yields a fundamental unit of any totally complex quartic order in $O\left(R D^{\varepsilon}\right)$ binary operations (for every $\varepsilon>0$ ), where $D$ is the absolute value of the discriminant and $R$ is the regulator of the order.

We establish an analogue of Galois' theorem on the symmetry of the continued fraction expansion of the square root of a rational number. We conclude the paper by presenting computational results for the orders $\mathbf{Z}[\sqrt[4]{-d}], 1 \leqslant d \leqslant 500$.

The author is indebted to the referee for many helpful corrections and suggestions.

2. Notations. In this paper

$L=\mathbf{Q}(\rho)$ is a totally complex quartic algebraic number field,

$\boldsymbol{\sigma}$ is a $\mathbf{Q}$-isomorphism of $L$ into $\mathbf{C}$, different from the complex conjugation. For $\xi \in L$ we write

$$
\xi^{(1)}=\xi, \quad \xi^{(2)}=\sigma(\xi), \quad \xi^{(3)}=\bar{\xi} \quad \text { and } \quad \xi^{(4)}=\overline{\sigma(\xi)} .
$$

$\mathcal{O}$ is an order of $L$,

$D$ is the absolute value of the discriminant of $\mathcal{O}$,

$R$ is the regulator of $\mathcal{O}$,

$\omega_{1}, \ldots, \omega_{4}$ is a $\mathbf{Z}$-basis of $\mathcal{O}$,

$\omega_{1}^{*}, \ldots, \omega_{4}^{*}$ is the corresponding dual basis,

$W=\max \left\{\left|\omega_{k}^{(i)}\right| \mid 1 \leqslant i, k \leqslant 4\right\}$,

$W^{*}=\max \left\{\left|\omega_{k}^{*(i)}\right| \mid 1 \leqslant i, k \leqslant 4\right\}$.

We assume that

$$
W \leqslant D^{1 / 2} \text {. }
$$

Such a basis can be computed using a basis reduction algorithm, e.g., [11], in the Minkowski lattice corresponding to $\mathcal{O}$. It follows immediately that

$$
W^{*} \leqslant 6 D \text {. }
$$

For a (fractional) ideal $a$ of $\mathcal{O}$ we fix

$$
d(\mathfrak{a})=\min \{d \in \mathbf{N} \mid d \mathfrak{a} \subseteq \mathcal{O}\}, \quad N(\mathfrak{a})=\text { norm of } \mathfrak{a} .
$$

3. The Method. We recall the main definitions and results of [3]. There, we introduced the map

$$
L \rightarrow \mathbf{R}^{2}, \quad \xi \rightarrow \xi=\left(|\xi|^{2},|\sigma(\xi)|^{2}\right)^{t}
$$

which, restricted to the multiplicative group $L^{\times}$, is a homomorphism, if we use the product

$$
\left(y_{1}, y_{2}\right)^{t} \cdot\left(y_{1}^{\prime}, y_{2}^{\prime}\right)^{t}=\left(y_{1} y_{1}^{\prime}, y_{2} y_{2}^{\prime}\right)^{t} \text {. }
$$

Moreover, $L^{\times}$acts on $\mathbf{L}$ by

$$
\xi^{*} \xi^{\prime}=\xi \xi^{\prime} \quad \text { for every } \xi \in L^{\times}, \xi^{\prime} \in \mathbf{L} .
$$

For a point $\vec{y}=\left(y_{1}, y_{2}\right)^{t} \in \mathbf{R}^{2}$ its norm is defined by

$$
N(\vec{y})=\left|y_{1} y_{2}\right| \text {. }
$$

Now let $a$ be a (fractional) ideal in $\mathcal{O}$. Then the image $a$ is a discrete set in $\mathbf{R}^{2}$. A point $0 \neq \vec{m}$ in $a$ is called a minimal point of $a$, if its norm body

$$
Q(\vec{m})=\left\{\vec{y} \in \mathbf{R}^{2} \mid 0 \leqslant y_{i} \leqslant m_{i} \text { for } 1 \leqslant i \leqslant 2\right\}
$$


does not contain points of $a$ aside from $\mathbf{0}$ and $\vec{m}$. Minimal points are of bounded norm

$$
N(\vec{m}) \leqslant\left(4 / \pi^{2}\right) D^{1 / 2} N(\mathfrak{a}) .
$$

Moreover, for $\{u, v\}=\{1,2\}$ the $u$-neighbor of a minimal point $\vec{m}$ is defined to be the (uniquely determined) minimal point $\vec{m}^{\prime}$ with $m_{u}^{\prime}<m_{v}$ and minimal $m_{u}^{\prime}$. For this neighbor we have by Minkowski's convex body theorem

$$
m_{u}^{\prime} m_{v} \leqslant\left(4 / \pi^{2}\right) D^{1 / 2} N(a) .
$$

Finally, 1 is a minimal point in $\mathcal{O}$ and all the minimal points of $\mathcal{O}$ can be arranged in a two-sided sequence $\left(\vec{m}_{k}\right)_{k \in \mathbf{Z}}, \vec{m}_{0}=\mathbf{1}$, where $\vec{m}_{k+1}$ is always the 2-neighbor of $\vec{m}_{k}$ and, in turn, $\vec{m}_{k}$ is the 1-neighbor of $\vec{m}_{k+1}$ for every $k \in \mathbf{Z}$. This sequence, called the GVA-expansion in $\mathcal{O}$, is of the purely periodic form

$$
\begin{gathered}
\ldots, \varepsilon^{-1 *} 1, \varepsilon^{-1 *} \vec{m}_{1}, \ldots, \varepsilon^{-1 *} \vec{m}_{p-1}, 1, \vec{m}_{1}, \ldots, \vec{m}_{p-1}, \\
\varepsilon^{*} \mathbf{1}, \varepsilon^{*} \vec{m}_{1}, \ldots, \varepsilon^{*} \vec{m}_{p-1}, \ldots
\end{gathered}
$$

Here $\varepsilon$ is a unit in $\mathcal{O}$, and if $p$ is chosen minimal, then $\varepsilon$ is a fundamental unit of $\mathcal{O}$, and $p$ is called the period length of the GVA in $\mathcal{O}$.

4. The Algorithm. Here is our algorithm for computing a fundamental unit $\varepsilon$ and the regulator $R$ of $\mathcal{O}$.

\section{Algorithm 4.1.}

Input: $\omega_{1}, \ldots, \omega_{4}$.

Output: $R, \varepsilon$.

1. Initialize: $k \leftarrow 0, N \leftarrow 1, \eta_{0} \leftarrow 1, a \leftarrow \mathcal{O}, R \leftarrow 0$.

2. Repeat until $k \geqslant 1$ and $N=1$ :

(a) $\mathfrak{a} \leftarrow\left(1 / \eta_{k}\right) \mathfrak{a}$.

(b) Compute $\eta_{k+1}$ in a such that $\eta_{\mathbf{k}+1}$ is the 2-neighbor of 1 in a.

(c) $k \leftarrow k+1, R \leftarrow R-\log \left|\eta_{k}\right|^{2}, N \leftarrow N \cdot\left|N_{L \mid \mathbf{Q}}\left(\eta_{k}\right)\right|$.

3. $\varepsilon \leftarrow \Pi_{j=0}^{k} \eta_{j}$.

Notice that we can compute a maximal system of pairwise nonassociated "minima" in the sense of [21, Section 3], if we calculate in step 2(b) all the $\eta$ in a such that $\eta$ is the 2-neighbor of 1 in $a$.

The representation of the principal ideals a will be discussed in Section 6 and the computation of $\eta_{k+1}$ will be described in Section 7 .

We conclude this section by giving a justification for our algorithm. We define for $0 \leqslant k \leqslant p$

$$
\mu_{k}=\prod_{j=0}^{k} \eta_{j}
$$

Then we have

$$
\mu_{k} \in \mathcal{O} \text { and } \mu_{\mathbf{k}}=\vec{m}_{k} \text { for } 0 \leqslant k \leqslant p
$$

and in step 2(b)

$$
\mathfrak{a}=\left(1 / \mu_{k}\right) \mathcal{O}
$$


In fact, (4.2) and (4.3) are true for $k=0$. Now suppose that (4.2) and (4.3) hold for $k \geqslant 0$. Since $\eta_{k+1} \in\left(1 / \mu_{k}\right) \mathcal{O}$, we must have $\mu_{k+1}=\mu_{k} \eta_{k+1} \in \mathcal{O}$. Moreover, $\eta_{\mathbf{k}+1}$ is the 2-neighbor of 1 in $\left(1 / \mu_{k}\right) \mathcal{O}$, and therefore $\mu_{k+1}=\mu_{k} \eta_{k+1}$ must be the 2-neighbor of $\mu_{\mathbf{k}}=\vec{m}_{k}$ in $\mathcal{O}$, i.e., $\mu_{\mathbf{k}+1}=\vec{m}_{k+1}$. It follows immediately that in step 2(c),

$$
N=N\left(\vec{m}_{k}\right)=\left|N_{L \mid \mathbf{Q}}\left(\vec{\mu}_{k}\right)\right| \text {. }
$$

If $k=p$, then by (3.3) and (4.4) we must have $N=1$, and because of the minimality of $p$, this is the first time that $N=1$ can happen.

5. The Theorem of Galois. By a theorem of Galois, the period of the continued fraction expansion of the square root of a positive rational number is symmetric, $\mathrm{cf}$. [14, Section 23]. A similar result is proved in this section. We assume that the order under consideration satisfies the condition

$$
\sigma(\mathcal{O})=\mathcal{O}
$$

This is true, for example, if $\mathcal{O}=\mathbf{Z}[\sqrt[4]{-d}], d \in \mathbf{N}$. Note that (5.1) implies that $L$ has a quadratic subfield. On the plane $\mathbf{R}^{2}$ we introduce the reflection

$$
\tilde{\boldsymbol{\sigma}}: \mathbf{R}^{2} \rightarrow \mathbf{R}^{2}, \quad \vec{y}=\left(y_{1}, y_{2}\right)^{t} \rightarrow \tilde{\boldsymbol{\sigma}}(\vec{y})=\left(y_{2}, y_{1}\right)^{t} .
$$

Then we have for every $\xi \in L$

$$
\sigma(\xi)=\tilde{\sigma}(\xi) .
$$

Consequently, the minimal points in $\mathcal{O}$ have the symmetry property:

Proposition 5.1. For every $k \in \mathbf{Z}$ we have $\vec{m}_{k}=\tilde{\sigma}\left(\vec{m}_{-k}\right)$.

Proof. Let $\vec{m}, \vec{m}^{\prime}$ be minimal points of $\mathcal{O}$. Then $\tilde{\sigma}(\vec{m})$ and $\sigma\left(\vec{m}^{\prime}\right)$ are minimal points of $\mathcal{O}$, and $\vec{m}$ is the 1-neighbor of $\vec{m}^{\prime}$ if and only if $\tilde{\sigma}(\vec{m})$ is the 2-neighbor of $\tilde{\sigma}\left(\vec{m}^{\prime}\right)$.

This yields the following application: If we have computed $\vec{m}_{0}, \ldots, \vec{m}_{k}$, then we know $\vec{m}_{-k}, \ldots, \vec{m}_{k}$. Hence, we can compute the regulator and a fundamental unit of $\mathcal{O}$ by computing only half the period of the GVA-expansion in $\mathcal{O}$. This is done by

\section{Algorithm 5.2.}

Input: $\omega_{1}, \ldots, \omega_{4}$.

Output: $R, \varepsilon$.

1. Initialize: $k \leftarrow 0, R \leftarrow 0, \eta_{0} \leftarrow 1, \mathfrak{a} \leftarrow \mathcal{O}$.

2. Repeat:
(a) $a \leftarrow\left(1 / \eta_{k}\right)$ a.
(b) Compute a complete system of nonassociated $\eta$ in a such that $\eta$ is the 2-neighbor of 1 in a. Choose one of these $\eta$ 's to be $\eta_{k+1}$.
(c) $R \leftarrow R-\log \left|\eta_{k+1}\right|^{2}$.
(d) If $\sigma(\mathfrak{a})=(1 / \eta) \mathfrak{a}$ holds for one of the $\eta$ 's of (b), then $\varepsilon \leftarrow \eta \Pi_{j=1}^{k}\left(\eta_{j} / \sigma\left(\eta_{j}\right)\right)$ and return.
(e) $R \leftarrow R+\log \left|\sigma\left(\eta_{k+1}\right)\right|^{2}$.
(f) If $\sigma\left(\left(1 / \eta_{k+1}\right) \mathfrak{a}\right)=(1 / \eta) \mathfrak{a}$ for one of the $\eta$ 's of (b), then $\varepsilon \leftarrow$ $\left(\eta / \sigma\left(\eta_{k+1}\right)\right) \prod_{j=1}^{k}\left(\eta_{j} / \sigma\left(\eta_{j}\right)\right)$, and return.
(g) $k \leftarrow k+1$. 
For the description of the representation of the ideals $a$ and the computation in 2(b), 2(d) and 2(f) we refer to Sections 6 and 7. We conclude this section by giving a justification of Algorithm 5.2.

For

$$
\mu_{k}=\prod_{j=0}^{k} \eta_{j}, \quad 0 \leqslant k \leqslant p,
$$

one can prove as in Section 4 that

$$
\mu_{k} \in \mathcal{O} \text { and } \mu_{\mathbf{k}}=\vec{m}_{k},
$$

and that in step 2(b), (d) and (f)

$$
a=\left(1 / \mu_{k}\right) \mathcal{O},
$$

and that

$$
\vec{m}_{k+1}=\eta * \mu_{\mathrm{k}}
$$

for every $\eta$ computed in step 2(b).

It follows by (5.1) and Proposition 5.1 that

$$
\sigma\left(\mu_{\mathbf{k}}\right)=\vec{m}_{-k}
$$

and that in step 2(d)

$$
\sigma(\mathfrak{a})=\left(1 / \sigma\left(\mu_{k}\right)\right) \mathcal{O}
$$

Now suppose that in step 2(d), $\sigma(\mathfrak{a})=(1 / \eta) \mathfrak{a}$. Since in 2(d)

$$
\varepsilon=\eta \mu_{k} / \sigma\left(\mu_{k}\right) \text {, }
$$

it follows from (5.4) and (5.7) that $\varepsilon$ is a unit in $\mathcal{O}$. Moreover, by (5.5) and (5.6),

$$
\varepsilon^{*} \vec{m}_{-k}=\vec{m}_{k+1} \text {. }
$$

Because of the minimality of the period length $p$, this can happen only if $p \mid 2 k+1$. But if $p=2 k+1$, then (5.9) holds for every fundamental unit $\varepsilon$ with $|\varepsilon|<1$. Let $\varepsilon$ be such a fundamental unit. It follows from (5.5), (5.6) and (5.9) that

$$
\varepsilon \cdot \sigma\left(\mu_{k}\right)=\alpha \cdot \eta_{k+1} \mu_{k}
$$

with $\alpha \in L, \alpha=1$. We set $\eta=\alpha \eta_{k+1}$ and see by (5.4), (5.7) and (5.10) that $\eta \in \mathfrak{a}$, $\eta=\eta_{k+1}$, and $\sigma(\mathfrak{a})=(1 / \eta) \mathfrak{a}$.

Analogously, one can show that in step 2(f) one has $\sigma\left(\left(1 / \eta_{k+1}\right) \mathfrak{a}\right)=(1 / \eta) \mathfrak{a}$ only if $\varepsilon$ is a unit and $p \mid 2 k+2$ and, in turn, that this happens in fact if $p=2 k+2$. Then $\varepsilon$ is again a fundamental unit of $\mathcal{O}$.

6. Basis Reduction and Ideal Representation. In this section we describe how we represent the ideals $a$ in Algorithm 4.1 and Algorithm 5.2.

First of all, we recall some properties of the basis reduction algorithm of Lenstra, Lenstra and Lovász [11]. Let $\vec{b}_{1}, \ldots, \vec{b}_{n}$ be a basis of a lattice $\Gamma$ in $\mathbf{Z}^{n}$ and let $B \geqslant 2$, $\left|\vec{b}_{j}\right| \leqslant B$ for $1 \leqslant j \leqslant n .^{* *}$ This algorithm yields in $O\left(n^{4} \log B\right)$ binary operations a basis $\vec{a}_{1}, \ldots, \vec{a}_{n}$ of $\Gamma$ which satisfies

$$
\prod_{j=1}^{n}\left|\vec{a}_{j}\right| \leqslant 2^{n(n-1) / 4} \operatorname{det}(\Gamma)
$$

where $\operatorname{det}(\Gamma)$ is the determinant of $\Gamma$.

${ }^{* *} \mid$ | denotes the Euclidean norm. 
Now let $a$ be an ideal in $\mathcal{O}$, and let $\alpha_{1}, \ldots, \alpha_{4}$ be a $\mathbf{Z}$-basis of $a$,

$$
\alpha_{j}=\left(\sum_{k=1}^{4} a_{k j} \omega_{k}\right) / d(a), \quad 1 \leqslant j \leqslant 4 .
$$

Denote by $\Gamma$ the lattice spanned by the columns of the integral matrix $A=\left(a_{k j}\right)$. We call $A$ an LLL-matrix of a, if the columns of $A$ form a basis of $\Gamma$ which is reduced in the sense of [11, p. 516]. The ideals in Algorithm 4.1 and Algorithm 5.2 are represented in terms of their common denominator and an LLL-matrix. It has turned out in our computational experience that these representing integers are always small compared to the discriminant of $a$. We are also able to give bounds on the denominators and the elements of the LLL-matrices. These bounds are polynomials in $D$, and this means that the number of digits of these integers is $O(\log D)$. This statement will be useful in our complexity analysis in Section 8 .

Proposition 6.1. If $A$ is an LLL-matrix of $\mathfrak{a}$, then the column vectors $\vec{a}_{1}, \ldots, \vec{a}_{4}$ of A satisfy

$$
c_{1}^{-1} d(\mathfrak{a}) N(\mathfrak{a})^{1 / 4} \leqslant\left|\vec{a}_{j}\right| \leqslant c_{2} d(\mathfrak{a}) N(\mathfrak{a})^{1 / 4} * * *
$$

for $1 \leqslant j \leqslant 4$.

Proof. Let $\alpha_{1}, \ldots, \alpha_{4}$ be defined as in (6.2). Then we have for $1 \leqslant j \leqslant 4$,

$$
\left|\boldsymbol{\alpha}_{j}^{(1)}\right|^{2}\left|\boldsymbol{\alpha}_{j}^{(2)}\right|^{2}=\left|N_{L \mid \mathbf{Q}}\left(\alpha_{j}\right)\right| \geqslant N(\mathfrak{a}) \text {. }
$$

Hence, we have $\left|\alpha_{j}^{(i)}\right| \geqslant N(\mathfrak{a})^{1 / 4}$ for $i=1$ or $i=2$, and the first inequality of (6.3) follows from (2.1), (6.2), and Cauchy's inequality, whereas the second one follows from the first one and (6.1), since in this case $\operatorname{det}(\Gamma)=N(\mathfrak{a})$.

COROLlaRy 6.2. If $A$ is an LLL-matrix of a and if $\alpha_{1}, \ldots, \alpha_{4}$ is the corresponding $\mathbf{Z}$-basis of $a$, defined in (6.2), then we have for $1 \leqslant i, j \leqslant 4$,

$$
\left|\alpha_{j}^{(i)}\right| \leqslant c_{3} N(a)^{1 / 4} \text {. }
$$

Proof. This corollary follows from (2.1), (6.2) and Proposition 6.1.

Corollary 6.3. If $a$ is one of the ideals, used in Algorithm 4.1 or Algorithm 5.2, if $A$ is an LLL-matrix of a with the columns $\vec{a}_{1}, \ldots, \vec{a}_{4}$, and if $\alpha_{1}, \ldots, \alpha_{4}$ is the corresponding Z-basis of a, defined in (6.2), then we have for $1 \leqslant i, j \leqslant 4$,

$$
\left|\vec{a}_{j}\right| \leqslant c_{4} \text { and }\left|\alpha_{j}^{(i)}\right| \leqslant c_{5} .
$$

Proof. It follows from (3.1), (4.2), (4.3), (5.3), and (5.4) that $N(\mathfrak{a}) \leqslant 1$ and $d(a) \leqslant D^{1 / 2}$.

The last result shows that the ideals $a$ in Algorithm 4.1 and Algorithm 5.2 can be represented by integral matrices which are-independently of $k$-of the same "small" size.

${ }^{* * *}$ The numbers $c_{k}, k \in \mathbf{N}$, are of the form $u D^{v}, u, v>0$. 
We finally remark that the comparison of the ideals in Algorithm 5.2 step 2(d) and 2(f) can be carried out by comparing the denominators and Hermite normal forms of the representation matrices.

7. The Neighbor Computation. In step 2(b) of Algorithm 4.1 we want to know a number $\eta$ in the ideal a such that $\eta$ is the 2-neighbor of $\mathbf{1}$ in a. Following the explanation given in Section 3, this means that we have to find $\eta$ in a with

$$
\left|\eta^{(1)}\right|^{2}<1 \text { and }\left|\eta^{(2)}\right|^{2} \leqslant\left(4 / \pi^{2}\right) D^{1 / 2} N(a)
$$

with minimal $\left|\eta^{(2)}\right|^{2}$.

Let $A=\left(a_{k, j}\right)_{1 \leqslant k, j \leqslant 4}$ be an LLL-matrix of $a$ and let $\alpha_{1}, \ldots, \alpha_{4}$ be the corresponding $\mathbf{Z}$-basis of $\mathfrak{a}$, defined in (6.2). For $\vec{x}=\left(x_{1}, \ldots, x_{4}\right)^{t} \in \mathbf{Z}^{4}$ and $1 \leqslant i \leqslant 2$ we write

$$
\eta^{(i)}(\vec{x})= \begin{cases}\sum_{j=1}^{4} x_{j} \alpha_{j}^{(i)}=\left(\sum_{k=1}^{4} \omega_{k}^{(i)} \sum_{j=1}^{4} a_{k j} x_{j}\right) / d(\mathfrak{a}) & \text { if } \vec{x} \neq \overrightarrow{0} \\ D^{1 / 4} N(\mathfrak{a})^{1 / 2} & \text { if } \vec{x}=\overrightarrow{0}\end{cases}
$$

Then we can compute $\eta$ using

PROCEDURE 7.1.

1. Initialize: $\vec{x}_{2} \leftarrow \overrightarrow{0}, f \leftarrow 2$.

2. Repeat:

Try to find $\vec{x}_{1} \in \mathbf{Z}^{4}$ satisfying

$$
\left|\eta^{(1)}\left(\vec{x}_{1}\right)\right|<1 \text { and }\left|\eta^{(2)}\left(\vec{x}_{1}\right)\right|<\left|\eta^{(2)}\left(\vec{x}_{2}\right)\right| / f \text {. }
$$

If the search is successful, then set $\vec{x}_{2} \leftarrow \vec{x}_{1}$, else

if $f=2$, then set $f \leftarrow 1$, else return $\eta=\eta^{(1)}\left(\vec{x}_{2}\right)$.

Notice that for all $\vec{x} \in \mathbf{Z}^{4}$ with

$$
\left|\eta^{(1)}(\vec{x})\right|<1 \text { and }\left|\eta^{(2)}(\vec{x})\right|<(2 / \pi) D^{1 / 4} N(a)^{1 / 2},
$$

by the well-known dual basis argument [2, p. 403],

$$
\left|\sum_{j=1}^{4} a_{k j} x_{j}\right| \leqslant 4 W^{*} d(a) D^{1 / 4} N(a)^{1 / 2}, \quad 1 \leqslant k \leqslant 4 .
$$

The comparisons in (7.3) have to be carried out using rational approximations $\hat{\omega}_{k}^{(i)}$ to $\omega_{k}^{(i)}, 1 \leqslant k, i \leqslant 4$. We must therefore discuss the question of how this is to be done. Let $\lambda>0$ have the property

$$
\max \left\{\left|\omega_{k}^{(i)}-\hat{\omega}_{k}^{(i)}\right| \mid 1 \leqslant i, k \leqslant 4\right\}<\lambda .
$$


For $\vec{x} \neq 0$, let $\hat{\eta}^{(i)}(\vec{x})$ be the approximation of $\eta^{(i)}(\vec{x})$ obtained by substituting $\omega_{k}^{(i)}$ by $\hat{\omega}_{k}^{(i)}$ in (7.2), and let $\hat{\eta}^{(2)}(0)$ be a rational approximation of $D^{1 / 4}$ such that $\left|\hat{\eta}^{(2)}(0)-D^{1 / 4}\right|<\lambda$. Finally, we set for $\vec{x} \in \mathbf{Z}^{4}$

$$
\delta_{1}(\vec{x})= \begin{cases}4 \lambda\left|\sum_{j=1}^{4} a_{k j} x_{j}\right| / d(a) & \text { if } \vec{x} \neq \overrightarrow{0} \\ \lambda & \text { if } \vec{x}=\overrightarrow{0}\end{cases}
$$

and

$$
\delta(\vec{x})=\delta_{1}(\vec{x})\left(2 D^{1 / 4}+\delta_{1}(\vec{x})\right) .
$$

Then it follows for every $\vec{x}$, subject to (7.4), that

$$
\delta(\vec{x}) \leqslant \delta=\delta_{1}\left(2 D^{1 / 4}+\delta_{1}\right)
$$

with

$$
\delta_{1}=16 \lambda W^{*} D^{1 / 4} N(\mathfrak{a})^{1 / 2},
$$

and we have for every $\vec{x} \in \mathbf{Z}^{4}$, subject to (7.4), and $1 \leqslant i \leqslant 2$,

$$
\left.|| \eta^{(i)}(\vec{x})\right|^{2}-\left|\hat{\eta}^{(i)}(\vec{x})\right|^{2} \mid \leqslant \delta(\vec{x}) \leqslant \delta .
$$

Hence, (7.3) can be true only if

$$
\left|\hat{\eta}^{(1)}\left(\vec{x}_{1}\right)\right|^{2}+\left|\hat{\eta}^{(2)}\left(\vec{x}_{1}\right)\right|^{2} \leqslant 1+\left|\hat{\eta}^{(2)}\left(\vec{x}_{2}\right)\right|^{2} / f^{2}+3 \delta
$$

The solutions of (7.10) can be computed using an algorithm of Fincke and Pohst [6] which yields all the integral solutions $\vec{x}$ of an inequality $Q(\vec{x}) \leqslant K$, where $Q$ is a positive definite $n$-dimensional rational quadratic form and $K \geqslant 0$ is a real constant. By (7.9) we are able to decide whether a solution of (7.10) satisfies (7.3) as long as

$$
\begin{aligned}
& \left.|| \hat{\eta}^{(1)}\left(\vec{x}_{1}\right)\right|^{2}-1 \mid>\delta\left(\vec{x}_{1}\right) \text { and } \\
& \left.|| \hat{\eta}^{(2)}\left(\vec{x}_{1}\right)\right|^{2}-\left|\hat{\eta}^{(2)}\left(\vec{x}_{2}\right)\right|^{2} / f^{2} \mid>\delta\left(\vec{x}_{1}\right)+\delta\left(\vec{x}_{2}\right) .
\end{aligned}
$$

This, in turn, is true if

$$
\left|\eta^{(1)}\left(\vec{x}_{1}\right)\right| \neq 1 \text { and }\left|\eta^{(2)}\left(\vec{x}_{1}\right)\right| \neq\left|\eta^{(2)}\left(\vec{x}_{2}\right)\right| / f
$$

and $\lambda$ is small enough. Notice that (7.12) can be tested by using [21, Proposition 2.2].

Concluding these remarks, we can carry out the search for $\vec{x}_{1}$ in Procedure 7.1 in the following way.

We enumerate the solutions of (7.10) using the algorithm of Fincke and Pohst [6]. If we find a solution $\vec{x}_{1}$, we check if $\vec{x}_{1}$ satisfies (7.4). If not, we reject $\vec{x}_{1}$ as a possible solution of (7.3). Otherwise, we check whether $\vec{x}_{1}$ is subject to

$$
\left|\hat{\eta}^{(1)}\left(\vec{x}_{1}\right)\right|^{2}<1-\delta\left(\vec{x}_{1}\right) \text { and }\left|\hat{\eta}^{(2)}\left(\vec{x}_{1}\right)\right|^{2}<\left|\hat{\eta}^{(2)}\left(\vec{x}_{2}\right)\right|^{2} / f^{2}-\delta\left(\vec{x}_{1}\right)-\delta\left(\vec{x}_{2}\right) \text {, }
$$


or

$$
\left|\hat{\eta}^{(1)}\left(\vec{x}_{1}\right)\right|^{2}>1+\delta\left(\vec{x}_{1}\right) \text { or }\left|\hat{\eta}^{(2)}\left(\vec{x}_{1}\right)\right|^{2}>\left|\hat{\eta}^{(2)}\left(\vec{x}_{2}\right)\right|^{2} / f^{2}+\delta\left(\vec{x}_{1}\right)+\delta\left(\vec{x}_{2}\right) .
$$

In the first case, we have found a solution of (7.3). In the second case, we must reject $\vec{x}_{1}$ as a possible solution of (7.3).

If neither the first nor the second case holds, a situation which we have never encountered in any of our computations, then we check whether (7.12) is true. If the answer is negative, then we must reject $\vec{x}_{1}$; otherwise, we have to increase the precision of our approximation to $\omega_{k}^{(i)}$, i.e., we have to decrease $\lambda$. During our computations we found the value $\lambda=10^{-12}$ always to be sufficient.

If we have enumerated all the solutions of (7.10) without finding a solution of (7.3), then no such solution exists.

In Algorithm 5.2 we need all the $\eta \in$ a such that $\eta$ is the 2-neighbor of 1 in a. These numbers can be computed by a further application of the algorithm in [6].

For our complexity analysis in the next section, it is necessary to be able to prove that there exists a value of $\lambda$ such that (7.11) follows from (7.12). This can be done analogously to the proof of [21, Proposition 4.1]. The result is

Proposition 7.12. We can choose $\lambda=c_{7}^{-1}$ and $\delta=c_{8} \lambda$ such that for every $\vec{x}_{1}, \vec{x}_{2}$ subject to (7.4) the following statements hold:

(i) If $\vec{x}_{2}=\overrightarrow{0}$, then it follows from

$$
\left|\eta^{(1)}\left(\vec{x}_{1}\right)\right|<1 \text { and }\left|\eta^{(2)}\left(\vec{x}_{1}\right)\right|<(2 / \pi) D^{1 / 4} N(a)^{1 / 2} / f
$$

that

$$
\left|\hat{\eta}^{(1)}\left(\vec{x}_{1}\right)\right|^{2} \leqslant 1-\delta \text { and }\left|\hat{\eta}^{(2)}\left(\vec{x}_{1}\right)\right|^{2} \leqslant\left|\hat{\eta}^{(2)}\left(\vec{x}_{2}\right)\right|^{2} / f^{2}-\delta
$$

Conversely, (7.14) implies (7.3).

(ii) Let $\vec{x}_{2} \neq 0$; then (7.14) and (7.3) are equivalent.

8. Complexity Analysis. It is well known that the continued fraction algorithm computes a fundamental unit of an order of a real quadratic field in $O\left(R^{\prime} D^{\prime \mu^{\prime}}\right)$ binary operations (for every $\mu^{\prime}>0$ ), where $R^{\prime}$ is the regulator and $D^{\prime}$ is the absolute value of the discriminants of the order, cf. [19]. An analogous result is true for Voronoi's generalized continued fraction algorithm in complex cubic fields, cf. [19]. The purpose of this section is to prove that Algorithm 4.1 and Algorithm 5.2 are of the same complexity.

Since by [4] the period length of the GVA in $\mathcal{O}$ is $O(R)$, also the number of iterations in Algorithm 4.1, step 2, is $O(R)$. Since we use LLL-matrices to represent the ideals $a$ in Algorithm 4.1, it follows from Corollary 6.3 and from (7.1) that step 2(a) requires $O\left(D^{\mu}\right)$ binary operations (for every $\mu>0$ ).

We must now analyze step 2(b), i.e., Procedure 7.1. Since 1 is a minimal point in $a$, the number of iterations, when $f=2$, must be $O(\log D)$. But then it follows from the same arguments as used in the proof of [4, Lemma 2] that the number of solutions of (7.3) with $f=1$ is $O(1)$. The number of iterations of the procedure is therefore $O(\log D)$. 
It remains to analyze the complexity of the search for $\vec{x}_{1}$ in Procedure 7.1. It follows from Proposition 7.12 that, instead of searching the convex body described by (7.3), which has irrational constraints, we can search the convex body described by (7.4) and (7.14), which has rational constraints. Though the algorithm of Fincke and Pohst [6] has turned out to be very efficient in practice, we cannot use the complexity analysis provided for this algorithm in [6]. Therefore, we replace this method by a procedure of Lenstra [12], which, however, is too complicated for practical implementation. This procedure solves our search problem in polynomial time in the size of the input data, because obviously, our convex set is "solvable", cf. [12, Remark (d) in Section 2]. In view of (2.1), (2.2), Corollary 6.3, (7.1), (7.5), and the choice of $\lambda$ in Proposition 7.12, the input data length is $O(\log D)$. Hence, Procedure 7.1 requires $O\left(D^{\mu}\right)$ binary operations (for every $\mu>0$ ).

Finally, it follows from (7.1) and the fact that by [4] the number of factors in step 3 of Algorithm 4.1 is $O(R)$, that $\varepsilon$ can be computed in $O\left(R D^{\mu}\right)$ steps (for every $\mu>0)$.

Concluding these remarks, we have

THEOREM 8.1. A fundamental unit of $\mathcal{O}$ can be computed by means of Algorithm 4.1 in $O\left(R D^{\mu}\right)$ binary operations ( for every $\mu>0$ ).

The same complexity can be proved for Algorithm 5.2.

9. Example. Let $\mathcal{O}=\mathbf{Z}[\rho], \rho=\sqrt[4]{-326}$. Since condition (5.1) is satisfied, we can apply Algorithm 5.2:

1. Initialization: $k \leftarrow 0, R \leftarrow 0, \eta_{0} \leftarrow 1, a \leftarrow \mathcal{O}$.

2. (a) $a \leftarrow \mathcal{O}$.

(b) $\eta=18+6 \rho+\rho^{2}$ is up to association the only number such that $\eta$ is the 2-neighbor of 1 in a, $\eta_{1} \leftarrow \eta$.

(c) $R \leftarrow 7.8633$.

(d) $\alpha_{1} \leftarrow 1, \alpha_{2} \leftarrow \rho, \alpha_{3} \leftarrow \rho^{2} / 2, \alpha_{4} \leftarrow \rho^{3} / 2,\left(1 / \eta_{1}\right) a=\oplus_{j=1}^{4} \mathbf{Z} \alpha_{j} \neq \sigma(a)$.

(e) $R \leftarrow 14.3402$.

(f) $\sigma\left(\left(1 / \eta_{1}\right) \mathfrak{a}\right)=\left(1 / \eta_{1}\right) \mathfrak{a}, \varepsilon \leftarrow \eta_{1} / \sigma\left(\eta_{1}\right)=1+108 \rho-36 \rho^{2}+6 \rho^{3}$.

10. Numerical Results. We have computed the GVA-expansions in the orders $\mathcal{O}=\mathbf{Z}[\sqrt[4]{-d}], d \in \mathbf{N}, d \neq 4 k^{4}$ for $1 \leqslant d \leqslant 500$. In Table 1 ,

$$
E_{1}+E_{2} \rho+E_{3} \rho^{2}+E_{4} \rho^{3}
$$

is a fundamental unit of $\mathcal{O}, \rho=\sqrt[4]{-d}$.

In Table 2 we denote by

PL the period length,

REG the regulator,

NR the relative norm of the fundamental units over $\mathbf{Z}[\sqrt{-d}]$. 


\section{TABLE 1}

Fundamental units of $\mathbf{Z}\left[(-d)^{1 / 4}\right], 1 \leqslant d \leqslant 40$.

\begin{tabular}{|c|c|c|c|c|}
\hline$D$ & $E_{1}$ & $E_{2}$ & $E_{3}$ & $E_{4}$ \\
\hline 1 & 0 & 1 & -1 & 1 \\
\hline 2 & -1 & 0 & 1 & -1 \\
\hline 3 & 2 & -2 & 1 & 0 \\
\hline 5 & -2 & 2 & -1 & 0 \\
\hline 6 & 1 & 4 & -4 & 2 \\
\hline 7 & 36 & -26 & 9 & 2 \\
\hline 8 & 1 & 2 & -2 & 1 \\
\hline 9 & -485 & 198 & 0 & -66 \\
\hline 10 & -27 & 12 & -1 & -3 \\
\hline 11 & -98 & 96 & -45 & 6 \\
\hline 12 & 23 & -14 & 4 & 1 \\
\hline 13 & -86 & 28 & 3 & -10 \\
\hline 14 & -13 & 2 & 2 & -2 \\
\hline 15 & 16 & 4 & -7 & 4 \\
\hline 16 & 577 & -204 & 0 & 51 \\
\hline 17 & -16 & 7 & -1 & -1 \\
\hline 18 & -13823 & 58332 & -36792 & 11512 \\
\hline 19 & 14439374 & 11821890 & -11320425 & 4956000 \\
\hline 20 & -9 & 6 & -2 & 0 \\
\hline 21 & 1 & 36 & -24 & 8 \\
\hline 22 & -91167 & 440140 & -267972 & 81146 \\
\hline 23 & 25899588 & -8909082 & 352849 & 1629810 \\
\hline 24 & -95 & 46 & -10 & -3 \\
\hline 25 & -4443 & 1405 & 0 & -281 \\
\hline 26 & -125 & 12 & 17 & -13 \\
\hline 27 & -1414178 & 220224 & 135531 & -126466 \\
\hline 28 & 57 & 2 & -12 & 7 \\
\hline 29 & 330206 & -411912 & 189709 & -39122 \\
\hline 30 & -74879 & 57396 & -21012 & 2218 \\
\hline 31 & 1975104 & 28184 & -371631 & 217672 \\
\hline 32 & 6913 & -360 & -1008 & 663 \\
\hline 33 & 67 & -74 & 32 & -6 \\
\hline 34 & -33 & -4 & 8 & -4 \\
\hline 35 & 64926 & -77790 & 34255 & -6768 \\
\hline 36 & 1351 & $\begin{array}{r}-390 \\
-390\end{array}$ & $\begin{array}{r}0 \\
290\end{array}$ & $\begin{array}{r}65 \\
\end{array}$ \\
\hline 37 & -571878 & 289258 & $\begin{array}{r}-71847 \\
\end{array}$ & $\begin{array}{r}-6356 \\
-54441051012990\end{array}$ \\
\hline $\begin{array}{l}38 \\
39\end{array}$ & -267790150327167 & $\begin{array}{r}-183064217333724 \\
-2544\end{array}$ & $\begin{array}{r}147714465189436 \\
840\end{array}$ & $\begin{array}{r}-54441051012990 \\
-68\end{array}$ \\
\hline 40 & $\begin{array}{r}3745 \\
-159\end{array}$ & $\begin{array}{r}-2544 \\
-94\end{array}$ & $\begin{array}{r}840 \\
78\end{array}$ & $\begin{array}{l}-20 \\
-29\end{array}$ \\
\hline
\end{tabular}

TABLE 2

Regulators of $\mathbf{Z}\left[(-d)^{1 / 4}\right]$

D PL NR REG

$\begin{array}{rrr}1 & 2 & 1 \\ 2 & 3 & -1 \\ 3 & 5 & 1 \\ 5 & 1 & -1 \\ 6 & 2 & 1 \\ 7 & 8 & 1 \\ 8 & 2 & 1 \\ 9 & 12 & 1 \\ 10 & 13 & -1 \\ 11 & 16 & 1 \\ 12 & 6 & 1 \\ 13 & 7 & -1 \\ 14 & 2 & 1\end{array}$

1.7627

2.4485

3.3258

3.5796

5.9660

8.9161

4.8969

13.7546

7.9923

11.7560

7.9666

10.3107

6.8013
D PL NR REG

$\begin{array}{rrrr}15 & 4 & 1 & 8.2853 \\ 16 & 10 & 1 & 14.1020 \\ 17 & 2 & 1 & 6.9942 \\ 18 & 20 & 1 & 25.3105 \\ 19 & 38 & 1 & 36.8971 \\ 20 & 2 & 1 & 6.4677 \\ 21 & 6 & 1 & 10.7870 \\ 22 & 26 & 1 & 29.4799 \\ 23 & 38 & 1 & 35.5300 \\ 24 & 6 & 1 & 10.7298 \\ 25 & 13 & -1 & 18.1845 \\ 26 & 9 & -1 & 11.4356 \\ 27 & 36 & 1 & 29.9319\end{array}$


TABLE 2 (continued)

\begin{tabular}{rrrr} 
D & PL & NR & \multicolumn{1}{c}{ REG } \\
& & & \\
28 & 8 & 1 & 10.2793 \\
29 & 23 & -1 & 29.1595 \\
30 & 36 & 1 & 25.0462 \\
31 & 28 & 1 & 31.1193 \\
32 & 10 & 1 & 19.5876 \\
33 & 4 & 1 & 11.9390 \\
34 & 4 & 1 & 9.4774 \\
35 & 28 & 1 & 25.9225 \\
36 & 14 & 1 & 15.8035 \\
37 & 27 & -1 & 28.3596 \\
38 & 70 & 1 & 70.3594 \\
39 & 16 & 1 & 18.9285 \\
40 & 10 & 1 & 13.8874 \\
41 & 9 & -1 & 11.5768 \\
42 & 14 & 1 & 20.9260 \\
43 & 72 & 1 & 65.4578 \\
44 & 58 & 1 & 44.6300 \\
45 & 28 & 1 & 30.5438 \\
46 & 6 & 1 & 10.6957 \\
47 & 62 & 1 & 65.8973 \\
48 & 8 & 1 & 13.3031 \\
49 & 40 & 1 & 47.6012 \\
50 & 35 & -1 & 38.7416 \\
51 & 26 & 1 & 29.8215 \\
52 & 16 & 1 & 19.2904 \\
53 & 21 & -1 & 24.6862 \\
54 & 104 & 1 & 96.5684 \\
55 & 10 & 1 & 19.9494 \\
56 & 4 & 1 & 8.1859 \\
57 & 12 & 1 & 21.0419 \\
58 & 11 & -1 & 14.3255 \\
59 & 120 & 1 & 120.9619 \\
60 & 4 & 1 & 9.6399 \\
61 & 19 & -1 & 25.0618 \\
62 & 4 & 1 & 11.0587 \\
63 & 2 & 1 & 6.2305 \\
65 & 1 & -1 & 6.2461 \\
66 & 2 & 1 & 11.1212 \\
67 & 104 & 1 & 114.9532 \\
68 & 2 & 1 & 9.7650 \\
69 & 40 & 1 & 42.9765 \\
70 & 36 & 1 & 43.1795 \\
71 & 64 & 1 & 75.4121 \\
72 & 2 & 1 & 8.4368 \\
73 & 14 & 1 & 26.4517 \\
74 & 9 & -1 & 13.7289 \\
75 & 48 & 1 & 54.9405 \\
76 & 22 & 1 & 34.8990 \\
77 & 10 & 1 & 14.0040 \\
78 & 44 & 1 & 53.3431 \\
79 & 116 & 1 & 143.0598 \\
80 & 4 & 1 & 14.3186 \\
81 & 52 & 1 & 63.4589 \\
82 & 15 & -1 & 17.2367 \\
83 & 112 & 1 & 100.7354 \\
84 & 20 & 1 & 20.6834 \\
85 & 33 & -1 & 33.3199 \\
86 & 126 & 1 & 103.5648 \\
87 & 8 & 1 & 13.9061 \\
& & &
\end{tabular}

D PL NR REG

$\begin{array}{rrrr}88 & 40 & 1 & 35.4889 \\ 89 & 44 & 1 & 54.5712 \\ 90 & 22 & 1 & 33.7471 \\ 91 & 34 & 1 & 50.8547 \\ 92 & 28 & 1 & 31.3328 \\ 93 & 56 & 1 & 60.0945 \\ 94 & 16 & 1 & 19.6221 \\ 95 & 32 & 1 & 37.4436 \\ 96 & 18 & 1 & 23.8641 \\ 97 & 30 & 1 & 34.3796 \\ 98 & 2 & 1 & 11.0465 \\ 99 & 26 & 1 & 49.2976 \\ 100 & 18 & 1 & 28.8727 \\ 101 & 13 & -1 & 23.6552 \\ 102 & 76 & 1 & 64.0178 \\ 103 & 186 & 1 & 174.6268 \\ 104 & 60 & 1 & 54.3461 \\ 105 & 8 & 1 & 16.5349 \\ 106 & 53 & -1 & 64.0716 \\ 107 & 106 & 1 & 121.6505 \\ 108 & 68 & 1 & 71.6995 \\ 109 & 27 & -1 & 44.3041 \\ 110 & 64 & 1 & 77.2438 \\ 111 & 10 & 1 & 22.1403 \\ 112 & 26 & 1 & 35.6645 \\ 113 & 12 & 1 & 20.0114 \\ 114 & 78 & 1 & 69.3097 \\ 115 & 116 & 1 & 103.6233 \\ 116 & 38 & 1 & 34.0075 \\ 117 & 28 & 1 & 31.8778 \\ 118 & 136 & 1 & 143.8799 \\ 119 & 126 & 1 & 131.9000 \\ 120 & 20 & 1 & 19.8271 \\ 121 & 22 & 1 & 23.9054 \\ 122 & 97 & -1 & 98.6025 \\ 123 & 10 & 1 & 26.3885 \\ 124 & 16 & 1 & 34.7878 \\ 125 & 9 & -1 & 17.8982 \\ 126 & 14 & 1 & 23.6952 \\ 127 & 166 & 1 & 187.6628 \\ 128 & 34 & 1 & 39.1752 \\ 129 & 72 & 1 & 79.2322 \\ 130 & 43 & -1 & 48.4233 \\ 131 & 96 & 1 & 107.5991 \\ 132 & 20 & 1 & 21.0849 \\ 133 & 76 & 1 & 88.0299 \\ 134 & 344 & 1 & 309.1082 \\ 135 & 16 & 1 & 31.3204 \\ 136 & 2 & 1 & 8.7844 \\ 137 & 3 & -1 & 10.6102 \\ 138 & 16 & 1 & 21.9224 \\ 139 & 272 & 1 & 282.8793 \\ 140 & 54 & 1 & 61.9243 \\ 141 & 2 & 1 & 9.1079 \\ 142 & 88 & 1 & 112.8690 \\ 143 & 68 & 1 & 101.5713 \\ 144 & 32 & 1 & 55.0184 \\ 145 & 3 & -1 & 11.6309 \\ 146 & 22 & 1 & 35.1488 \\ & & & \end{array}$


TABLE 2 (continued)

\begin{tabular}{|c|c|c|c|c|c|c|c|}
\hline D & PL & NR & REG & D & PL & NR & REG \\
\hline 147 & 38 & 1 & 54.8322 & 206 & 44 & 1 & 50.1061 \\
\hline 148 & 42 & 1 & 55.1209 & 207 & 8 & 1 & 12.2720 \\
\hline 149 & 189 & -1 & 194.0644 & 208 & 14 & 1 & 20.6215 \\
\hline 150 & 34 & 1 & 52.7798 & 209 & 120 & 1 & 116.8674 \\
\hline 151 & 40 & 1 & 53.3334 & 210 & 74 & 1 & 74.1316 \\
\hline 152 & 42 & 1 & 51.3585 & 211 & 184 & 1 & 204.1566 \\
\hline 153 & 34 & 1 & 56.7027 & 212 & 86 & 1 & 85.6995 \\
\hline 154 & 36 & 1 & 51.5545 & 213 & 40 & 1 & 55.5989 \\
\hline 155 & 4 & 1 & 13.8075 & 214 & 250 & i & 252.4171 \\
\hline 156 & 2 & 1 & 8.5164 & 215 & 158 & 1 & 179.6845 \\
\hline 157 & 105 & -1 & 98.3062 & 216 & 48 & $i$ & 53.6943 \\
\hline 158 & 18 & 1 & 29.2540 & 217 & 8 & 1 & 15.4263 \\
\hline 159 & 140 & 1 & 140.2070 & 218 & 53 & -1 & 61.8690 \\
\hline 160 & 56 & 1 & 63.9380 & 219 & 16 & 1 & 23.9217 \\
\hline 161 & 10 & 1 & 17.0583 & 220 & 20 & 1 & 25.9591 \\
\hline 162 & 168 & 1 & 176.2886 & 221 & 20 & 1 & 31.5731 \\
\hline 163 & 248 & 1 & 255.1354 & 222 & 12 & 1 & 29.2041 \\
\hline 164 & 36 & 1 & 42.4639 & 223 & 256 & 1 & 287.3029 \\
\hline 165 & 46 & 1 & 45.3833 & 224 & 12 & 1 & 27.2051 \\
\hline 166 & 224 & 1 & 226.4442 & 225 & 68 & 1 & 92.6691 \\
\hline 167 & 258 & 1 & 262.0549 & 226 & 2 & 1 & 12.2875 \\
\hline 168 & 24 & 1 & 28.3465 & 227 & 292 & 1 & 300.2000 \\
\hline 169 & 39 & -1 & 60.1234 & 228 & 30 & 1 & 37.5111 \\
\hline 170 & 57 & -1 & 57.0557 & 229 & 7 & -1 & 18.6889 \\
\hline 171 & 30 & 1 & 45.0703 & 230 & 114 & 1 & 137.2790 \\
\hline 172 & 12 & 1 & 29.3731 & 231 & 8 & 1 & 20.7683 \\
\hline 173 & 165 & -1 & 151.4328 & 232 & 70 & 1 & 82.9100 \\
\hline 174 & 188 & 1 & 195.8745 & 233 & 92 & 1 & 94.4689 \\
\hline 175 & 12 & 1 & 23.1631 & 234 & 14 & 1 & 25.1475 \\
\hline 176 & 14 & 1 & 23.5120 & 235 & 154 & 1 & 162.1329 \\
\hline 177 & 6 & 1 & 14.8157 & 236 & 106 & 1 & 120.1679 \\
\hline 178 & 40 & 1 & 56.5423 & 237 & 188 & 1 & 161.0306 \\
\hline 179 & 430 & 1 & 436.3769 & 238 & 48 & 1 & 45.0959 \\
\hline 180 & 22 & 1 & 31.3516 & 239 & 88 & 1 & 95.7492 \\
\hline 181 & 71 & -1 & 93.3738 & 240 & 8 & 1 & 16.5705 \\
\hline 182 & 48 & 1 & 42.8558 & 241 & 50 & $i$ & 67.9555 \\
\hline 183 & 46 & 1 & 59.6027 & 242 & 322 & 1 & 355.5986 \\
\hline 184 & 52 & 1 & 51.7444 & 243 & 270 & 1 & 269.3875 \\
\hline 185 & 4 & 1 & 11.7772 & 244 & 134 & 1 & 145.6830 \\
\hline 186 & 108 & 1 & 114.2256 & 245 & 206 & 1 & 194.9075 \\
\hline 187 & 134 & 1 & 147.8083 & 246 & 114 & 1 & 107.766 \\
\hline 188 & 8 & 1 & 20.1458 & 247 & 174 & 1 & 177.9984 \\
\hline 189 & 50 & 1 & 60.6175 & 248 & 22 & 1 & 32.0201 \\
\hline 190 & 26 & 1 & 43.3695 & 249 & 116 & 1 & 142.7931 \\
\hline 191 & 164 & 1 & 190.6173 & 250 & 159 & -1 & 173.5928 \\
\hline 192 & 44 & 1 & 63.7329 & 251 & 318 & l & 334.1780 \\
\hline 193 & 50 & 1 & 71.7403 & 252 & 32 & 1 & 41.529 \\
\hline 194 & 108 & 1 & 118.2099 & 253 & 34 & 1 & 49.4254 \\
\hline 195 & 40 & 1 & 49.7549 & 254 & 4 & 1 & 13.2797 \\
\hline 196 & 62 & 1 & 77.5225 & 255 & 70 & 1 & 78.7884 \\
\hline 197 & 155 & -1 & 128.6433 & 256 & 90 & 1 & 112.8158 \\
\hline 198 & 12 & 1 & 15.4730 & 257 & 13 & -1 & 19.5379 \\
\hline 199 & 170 & 1 & 162.9320 & 258 & 74 & $i$ & 83.6537 \\
\hline 200 & 76 & 1 & 77.4831 & 259 & 96 & 1 & 89.4168 \\
\hline 201 & 38 & 1 & 47.7578 & 260 & 12 & 1 & 23.0330 \\
\hline 202 & 147 & -1 & 151.4960 & 261 & 28 & $i$ & 33.4639 \\
\hline 203 & 38 & 1 & 38.4180 & 262 & 360 & $i$ & 388.5790 \\
\hline 204 & 86 & 1 & 89.9429 & 263 & 94 & 1 & 106.1759 \\
\hline 205 & 56 & 1 & 46.7214 & 264 & 10 & $i$ & 18.1441 \\
\hline
\end{tabular}


TABLE 2 (continued)

D PL NR REG

$\begin{array}{rrrr}265 & 46 & 1 & 53.4454 \\ 266 & 42 & 1 & 52.5164 \\ 267 & 174 & 1 & 171.0466 \\ 268 & 50 & 1 & 65.1967 \\ 269 & 273 & -1 & 273.0119 \\ 270 & 62 & 1 & 68.0688 \\ 271 & 128 & 1 & 108.5475 \\ 272 & 40 & 1 & 55.9533 \\ 273 & 28 & 1 & 45.2243 \\ 274 & 4 & 1 & 13.9617 \\ 275 & 90 & 1 & 100.3821 \\ 276 & 8 & 1 & 15.6401 \\ 277 & 217 & -1 & 226.3740 \\ 278 & 314 & 1 & 355.4098 \\ 279 & 38 & 1 & 46.5751 \\ 280 & 40 & 1 & 46.5511 \\ 281 & 38 & 1 & 53.5975 \\ 282 & 64 & 1 & 78.7796 \\ 283 & 498 & 1 & 534.2976 \\ 284 & 128 & 1 & 160.7741 \\ 285 & 4 & 1 & 13.3667 \\ 286 & 116 & 1 & 136.5902 \\ 287 & 150 & 1 & 165.4356 \\ 288 & 14 & 1 & 25.3105 \\ 289 & 118 & 1 & 144.4419 \\ 290 & 147 & -1 & 151.2632 \\ 291 & 18 & 1 & 26.7751 \\ 292 & 46 & 1 & 52.1155 \\ 293 & 271 & -1 & 217.7830 \\ 294 & 288 & 1 & 314.4971 \\ 295 & 16 & 1 & 33.6647 \\ 296 & 86 & 1 & 95.7995 \\ 297 & 60 & 1 & 81.3816 \\ 298 & 83 & -1 & 71.4501 \\ 299 & 86 & 1 & 92.5525 \\ 300 & 8 & 1 & 18.5757 \\ 301 & 26 & 1 & 36.0290 \\ 302 & 216 & 1 & 218.2099 \\ 303 & 232 & 1 & 235.1681 \\ 304 & 134 & 1 & 147.5885 \\ 305 & 51 & -1 & 67.4837 \\ 306 & 18 & 1 & 29.6483 \\ 307 & 482 & 1 & 490.9257 \\ 308 & 12 & 1 & 20.2496 \\ 309 & 370 & 1 & 368.9610 \\ 310 & 62 & 1 & 64.4781 \\ 311 & 94 & 1 & 120.4224 \\ 312 & 4 & 1 & 10.7129 \\ 313 & 61 & -1 & 87.0739 \\ 314 & 11 & -1 & 18.2544 \\ 315 & 20 & 1 & 33.8935 \\ 316 & 8 & 1 & 23.0731 \\ 317 & 79 & -1 & 80.5413 \\ 318 & 4 & 1 & 12.1182 \\ 319 & 58 & 1 & 72.4672 \\ 320 & 4 & 1 & 12.9354 \\ 321 & 4 & 1 & 13.5139 \\ 322 & 4 & 1 & 14.3279 \\ 323 & 2 & 1 & 7.8586\end{array}$

D PL NR REG

$\begin{array}{rrrr}325 & 1 & -1 & 7.8617 \\ 326 & 2 & 1 & 14.3402 \\ 327 & 2 & 1 & 13.5324 \\ 328 & 2 & 1 & 12.9601 \\ 329 & 222 & 1 & 222.7142 \\ 330 & 2 & 1 & 12.1552 \\ 331 & 166 & 1 & 200.8754 \\ 332 & 4 & 1 & 23.1719 \\ 333 & 10 & 1 & 34.0602 \\ 334 & 126 & 1 & 154.5054 \\ 335 & 240 & 1 & 272.3563 \\ 336 & 2 & 1 & 10.7870 \\ 337 & 16 & 1 & 36.3692 \\ 338 & 9 & -1 & 17.7100 \\ 339 & 270 & 1 & 307.0294 \\ 340 & 10 & 1 & 20.4472 \\ 341 & 190 & 1 & 194.7969 \\ 342 & 6 & 1 & 29.9819 \\ 343 & 454 & 1 & 436.8904 \\ 344 & 76 & 1 & 78.8263 \\ 345 & 56 & 1 & 59.2368 \\ 346 & 49 & -1 & 61.1623 \\ 347 & 122 & 1 & 160.9133 \\ 348 & 4 & 1 & 18.8724 \\ 349 & 257 & -1 & 304.3413 \\ 350 & 144 & 1 & 152.6403 \\ 351 & 46 & 1 & 82.8841 \\ 352 & 80 & 1 & 82.0567 \\ 353 & 78 & 1 & 80.3032 \\ 354 & 148 & 1 & 179.1556 \\ 355 & 56 & 1 & 70.2960 \\ 356 & 52 & 1 & 53.3035 \\ 357 & 144 & 1 & 123.0712 \\ 358 & 616 & 1 & 646.7992 \\ 359 & 28 & 1 & 35.6185 \\ 360 & 6 & 1 & 25.9792 \\ 361 & 120 & 1 & 163.5475 \\ 362 & 215 & -1 & 222.2816 \\ 363 & 66 & 1 & 75.3363 \\ 364 & 182 & 1 & 191.6409 \\ 365 & 73 & -1 & 100.3562 \\ 366 & 134 & 1 & 148.8015 \\ 367 & 470 & 1 & 420.3721 \\ 368 & 114 & 1 & 142.1201 \\ 369 & 66 & 1 & 103.1073 \\ 370 & 189 & -1 & 181.5445 \\ 371 & 36 & 1 & 38.7761 \\ 372 & 10 & 1 & 16.2358 \\ 373 & 168 & 1 & 166.4816 \\ 374 & 168 & 1 & 161.2338 \\ 375 & 232 & 1 & 261.0032 \\ 376 & 12 & 1 & 26.1178 \\ 377 & 57 & -1 & 59.7611 \\ 378 & 38 & 1 & 71.0890 \\ 379 & 820 & 1 & 865.4943 \\ 380 & 24 & 1 & 30.6399 \\ 381 & 218 & 1 & 223.3955 \\ 382 & 215 & -1 & 256.2240 \\ 383 & 502 & 1 & 524.9965\end{array}$


TABLE 2 (continued)

\begin{tabular}{|c|c|c|c|c|c|}
\hline PL & REG & $\mathrm{D}$ & PL & NR & REG \\
\hline 384 & 85.8386 & 443 & 158 & 1 & \\
\hline 385 & 76.2725 & 444 & 50 & $\begin{array}{l}1 \\
1\end{array}$ & $\begin{array}{r}185.2916 \\
54.7087\end{array}$ \\
\hline 386 & 19.7568 & 445 & 16 & 1 & 34.7171 \\
\hline 387 & 42.9218 & 446 & 22 & I & 38.3673 \\
\hline 388 & 68.9839 & 447 & 52 & I & 62.8728 \\
\hline 389 & 88.9151 & 448 & 68 & 1 & 82.2346 \\
\hline 390 & 33.6724 & 449 & 50 & 1 & 88.9369 \\
\hline 162 & 171.7357 & 450 & 34 & I & 37.2955 \\
\hline 392 & 77.3254 & 451 & 144 & 1 & 158.3658 \\
\hline 393 & 89.0531 & 452 & 8 & 1 & 22.1945 \\
\hline 493 & 541.8796 & 453 & 308 & 1 & 266.8515 \\
\hline 395 & 43.7689 & 454 & 1062 & 1 & 1211.1332 \\
\hline 396 & 44.2289 & 455 & 188 & 1 & 187.0916 \\
\hline 260 & 248.2283 & 456 & 12 & 1 & 21.6822 \\
\hline 398 & 13.9520 & 457 & 41 & -1 & 50.0053 \\
\hline 399 & 41.2984 & 458 & 206 & 1 & 229.7405 \\
\hline 108 & 145.4757 & 459 & 448 & 1 & 491.2462 \\
\hline 401 & 99.6167 & 460 & 156 & 1 & 159.9725 \\
\hline 402 & 66.0425 & 461 & 71 & -1 & 73.7591 \\
\hline 208 & 265.5586 & 462 & 126 & 1 & 114.3615 \\
\hline 160 & 215.3186 & 463 & 778 & 1 & 766.1231 \\
\hline 236 & 257.7341 & 464 & 42 & 1 & 58.3189 \\
\hline 406 & 43.1812 & 465 & 6 & 1 & 16.5710 \\
\hline 407 & 59.3021 & 466 & 24 & 1 & 31.8975 \\
\hline 408 & 58.9399 & 467 & 428 & 1 & 471.6362 \\
\hline 223 & 275.1346 & 468 & 30 & 1 & 36.9668 \\
\hline 410 & 81.7698 & 469 & 226 & 1 & 274.8130 \\
\hline 411 & 76.6110 & 470 & 352 & 1 & 371.6888 \\
\hline 142 & 152.9192 & 471 & 82 & $i$ & 83.2163 \\
\hline 334 & 288.8929 & 472 & 164 & 1 & 190.5559 \\
\hline 206 & 223.2892 & 473 & 148 & 1 & 176.9863 \\
\hline 324 & 306.4599 & 474 & 72 & i & 94.1183 \\
\hline 82 & 91.4846 & 475 & 66 & 1 & 100.7383 \\
\hline 32 & 46.7187 & 476 & 10 & 1 & 22.9074 \\
\hline 124 & 151.9582 & 477 & 284 & 1 & 294.5090 \\
\hline 104 & 92.2576 & 478 & 14 & 1 & 25.804 .2 \\
\hline 38 & 41.1450 & 479 & 364 & 1 & 361.1309 \\
\hline 18 & 23.1981 & 480 & 86 & $\overline{1}$ & 100.1847 \\
\hline 562 & 566.4572 & 481 & 51 & -1 & 65.8861 \\
\hline 34 & 38.9541 & 482 & 88 & 1 & 116.3825 \\
\hline 176 & 193.3631 & 483 & 78 & 1 & 76.4443 \\
\hline 50 & 66.1080 & 484 & 108 & 1 & 119.7289 \\
\hline 116 & 133.3298 & 485 & 161 & -1 & 183.4538 \\
\hline 232 & 256.8214 & 486 & 294 & 1 & 311.9160 \\
\hline 422 & 401.2819 & 487 & 184 & 1 & 185.0248 \\
\hline 14 & 30.5790 & 488 & 174 & $i$ & 156.7782 \\
\hline 28 & 35.7034 & 489 & 264 & 1 & 277.8649 \\
\hline 468 & 436.4574 & 490 & 220 & 1 & 274.5838 \\
\hline 38 & 59.8639 & 491 & 566 & 1 & 559.1352 \\
\hline 168 & 175.7602 & 492 & 188 & 1 & 190.6584 \\
\hline 8 & 25.3625 & 493 & 203 & -1 & 197.4414 \\
\hline 128 & 131.6412 & 494 & 244 & 1 & 286.6531 \\
\hline 70 & 103.3975 & 495 & 46 & 1 & 66.3733 \\
\hline 376 & 329.6845 & 496 & 50 & $i$ & 62.2386 \\
\hline 64 & 69.6490 & 497 & 6 & 1 & 19.6465 \\
\hline 434 & 388.0275 & 498 & 154 & 1 & 204.6873 \\
\hline 40 & 68.4719 & 499 & 46 & $i$ & 79.0547 \\
\hline 40 & 52.1058 & 500 & 140 & I & 161.6921 \\
\hline
\end{tabular}


Notice that NR $=-1$ if and only if PL is odd for $5 \leqslant d \leqslant 500$.

The computations were carried out on the CDC-Cyber 76 of the Universität zu Köln and the VAX 11/785 of the Department of Electrical Engineering of The Ohio State University.

Department of Mathematics

The Ohio State University

Columbus, Ohio 43210

1. H. Amara, "Groupe des classes et unité fondamentale des extensions quadratiques relatives à un corps quadratique imaginaire principal," Pacific J. Math., v. 96, 1981, pp. 1-12.

2. Z. I. Borevich \& I. R. Shafarevich, Number Theory, Academic Press, New York, 1966.

3. J. BuchmanN, “A generalization of Voronoi's unit algorithm I, II," J. Number Theory, v. 20, 1985, pp. 177-209.

4. J. BuchmanN, “Abschätzung der Periodenlänge einer verallgemeinerten Kettenbruchentwicklung," J. Reine Angew. Math., v. 361, 1985, pp. 27-34.

5. B. N. Delone \& D. K. FaddeEv, The Theory of Irrationalities of the Third Degree, Transl. Math. Monographs, vol. 10, Amer. Math. Soc., Providence, R. I., 1964.

6. U. FINCKE \& M. POHST, "Improved methods for calculating vectors of short length, including a complexity analysis," Math. Comp., v. 44, 1985, pp. 463-471.

7. N. Jeans, "Calculation of fundamental units in some types of quartic number fields," Bull. Austral. Math. Soc., v. 31, 1985, pp. 479-480.

8. T. KuвOTA, "Über den bizyklischen, biquadratischen Zahlkörper," Nagoya Math. J., v. 10, 1956, pp. $65-85$.

9. R. B. LaKeIN, "Computation of the ideal class group of certain complex quartic fields," Math. Comp., v. 28, 1974, pp. 839-846.

10. R. B. Lakein, "Computation of the ideal class groups of certain complex quartic fields. II," Math. Comp., v. 29, 1975, pp. 137-144.

11. A. K. Lenstra, H. W. Lenstra, JR. \& L. Lovász, "Factoring polynomials with rational coefficients," Math. Ann., v. 261, 1982, pp. 515-534.

12. H. W. LeNSTRA, JR., "Integer programming with a fixed number of variables," Math. Oper. Res., v. 8,1983 , pp. 538-548.

13. H. W. Lenstra, JR., On the Calculation of Regulators and Class Numbers of Quadratic Fields (Proc. Journées Arithmétiques 1980, Exeter), London Math. Soc. Lecture Notes Ser., vol. 56, 1982, pp. 123-150.

14. O. PerRon, Die Lehre von den Kettenbrüchen, Teubner, Stuttgart, 1954.

15. R. Scharlau, "The fundamental unit in quadratic extensions of imaginary quadratic number fields," Arch. Math., v. 34, 1980, pp. 534-537.

16. D. Shanks, The Infrastructure of a Real Quadratic Field and Its Applications, Proc. 1972 Number Theory Conf., Boulder, Colorado, 1973, pp. 217-224.

17. R. P. Steiner, On the Units in Algebraic Number Fields, Proc. 6th Manitoba Conf. Numer. Math., 1976, pp. 413-435.

18. G. VoronoI, A Generalization of the Continued Fraction Algorithm, Dissertation, Warsaw, 1896.

19. H. C. Williams, "Continued fractions and number-theoretic computations," Rocky Mountain J. Math., v. 15, 1985, pp. 621-655.

20. H. C. Williams, G. W. DuecK \& B. K. SChmid, "A rapid method of evaluating the regulator and the class number of a pure cubic field," Math. Comp., v. 41, 1983, pp. 235-286.

21. J. Buchmann \& H. C. Williams, "On principal ideal testing in totally complex quartic fields and the determination of certain cyclotomic constants," Math. Comp., v. 48, 1987, pp. 55-66.

22. H. G. Zimmer, Computational Problems, Methods and Results in Algebraic Number Theory, Lecture Notes in Math., vol. 262, Springer-Verlag, Berlin and New York, 1972. 